\title{
A wider role for polyamines in biofilm formation
}

\author{
Ece Karatan • Anthony J. Michael
}

Received: 8 June 2013/Accepted: 17 June 2013/Published online: 24 July 2013

(C) Springer Science+Business Media Dordrecht 2013

\begin{abstract}
Polyamines play an essential role in biofilm formation of diverse Gram-negative and Grampositive bacteria. Biosynthetic pathways and transport systems for diverse polyamines have been identified as key components of bacterial biofilm formation.
\end{abstract}

Keywords Bacillus subtilis · Biofilm ·

Norspermidine $\cdot$ Polyamine $\cdot$ Spermidine .

Vibrio cholerae

A recent paper published in Biotechnology Letters (Zhang et al. 2013) reported the interesting finding that the substrate-binding protein, PotD, of the Escherichia coli spermidine-preferential polyamine uptake transporter stimulates biofilm formation. Surprisingly, the authors appeared to be unaware of other published work, or at least did not reference such work, demonstrating a role for PotD and polyamines in biofilm formation. To redress this oversight we provide here a brief overview of the role of polyamines in biofilm formation and disassembly.

E. Karatan

Department of Biology, Appalachian State University, Boone, NC 28608, USA

e-mail: karatane@appstate.edu

\section{A. J. Michael ( $\square)$}

Department of Pharmacology, University of Texas Southwestern Medical Center, Dallas, TX 75390, USA

e-mail: anthony.michael@utsouthwestern.edu
Polyamines are linear, organic polycations that are fully protonated at physiological $\mathrm{pH}$ and are found in most bacteria. Whereas the asymmetrical polyamine, spermidine $\mathrm{H}_{2} \mathrm{~N}\left(\mathrm{CH}_{2}\right)_{3} \mathrm{NH}\left(\mathrm{CH}_{2}\right)_{4} \mathrm{NH}_{2}$, is widespread in bacteria, the shorter symmetrical version norspermidine, $\mathrm{H}_{2} \mathrm{~N}\left(\mathrm{CH}_{2}\right)_{3} \mathrm{NH}\left(\mathrm{CH}_{2}\right)_{3} \mathrm{NH}_{2}$, is limited mainly to Vibrio species and extreme hyperthermophiles (Hamana and Matsuzaki 1992). It is not known if there is a common function of polyamines in bacteria but recently a role for polyamines in biofilm formation was identified in the cholera agent Vibrio cholerae (Karatan et al. 2005; Lee et al. 2009), the plague bacterium Yersinia pestis (Patel et al. 2006), the model Gram-positive species Bacillus subtilis (Burrell et al. 2010), and in E. coli (Sakamoto et al. 2012) and Neisseria gonorrhoeae (Goytia et al. 2013). Biofilms are multicellular communities of bacteria encased in an extracellular matrix of exopolysaccharide, protein and sometimes DNA. In $V$. cholerae, extracellular norspermidine enhances biofilm formation but elimination of intracellular norspermidine by mutation of genes encoding norspermidine biosynthetic enzymes leads to inhibition of biofilm formation. This defect of biofilm formation is rescued by provision of exogenous norspermidine that is imported into the cell (Lee et al. 2009). Exogenous norspermidine enhances biofilm formation in $V$. cholerae by a mechanism that is dependent on the presence of two proteins, NspS and MbaA. NspS is homologous to PotD and PotD1, periplasmic substrate-binding proteins of E. coli and $V$. cholerae ABC-type transporters for spermidine, 
whereas MbaA is predicted to be an integral membrane protein that also contains GGDEF and EAL domains, and therefore is likely to be involved in signal transduction using the second messenger cyclicdi-GMP (Karatan et al. 2005). The same system is also responsible for responding to exogenous spermidine, which inhibits biofilm formation (McGinnis et al. 2009). In addition, deletion of the potDl gene in $V$. cholerae results in a much higher propensity to form biofilms, indicating that PotD1 is a repressor of biofilm formation (McGinnis et al. 2009). Under most conditions, V. cholerae does not synthesize any appreciable amount of spermidine, and therefore the potD1 mutants are devoid of this polyamine. Whether PotD1 directly inhibits biofilm formation or its effect is an indirect consequence of elimination of intracellular spermidine is currently unknown. In summary, norspermidine enhances $V$. cholerae biofilm formation whereas spermidine represses it both as extracellular signals and as intracellular metabolites.

Mutants of $Y$. pestis lacking both speA and speC genes, encoding enzymes in two different biosynthetic pathways for the spermidine precursor putrescine, are unable to form biofilms (Patel et al. 2006). This defect can be rescued by exogenous putrescine that is imported into the cell whereas exogenous spermidine cannot be taken up (Wortham et al. 2010). Because Y. pestis is able to synthesize spermidine using putrescine, it is equally likely that spermidine rather than putrescine directly affects biofilm formation. Absence of putrescine and spermidine also leads to a large reduction in the levels of the hemin storage (Hms) proteins, HmsR, $\mathrm{HmsS}$, and HmsT, which are necessary for biofilm formation (Wortham et al. 2010). HmsR and HmsS are involved in the production of the biofilm polysaccharide $\beta-1,6-N$-acetylglucosamine and HmsT is an integral membrane diguanylate cyclase that can synthesize cyclic-di-GMP. Thus, polyamines exert their effect on $Y$. pestis biofilms in part by maintaining adequate production of several key proteins involved in biofilm formation.

Two types of biofilm are formed by the model Gram-positive species B. subtilis: those formed on solid surfaces are known as colony biofilms and are characterised by a wrinkled morphology; those formed at a liquid-air interface are known as pellicles. Genetic abrogation of spermidine biosynthesis in B. subtilis significant decreases both colony and pellicle biofilm formation that are then restored by provision of exogenous spermidine (Burrell et al. 2010). Similarly, a mutant of putrescine and spermidine biosynthesis in E. coli also reduces biofilm formation (Sakamoto et al. 2012). Formation of biofilms in B. subtilis, Staphylococcus aureus and $E$. coli was found to be inhibited by exogenously supplied norspermidine (Kolodkin-Gal et al. 2012), whereas the tetraamine spermine but not norspermidine inhibited biofilm formation in Neisseria gonorrhoeae (Goytia et al. 2013).

In conclusion, polyamines act on different aspects of biofilm formation, both extracellularly and intracellularly. Norspermidine plays an opposite role in biofilm formation in $V$. cholerae, where it promotes biofilm formation, compared to in $B$. subtilis, where it is inhibitory. So far, polyamines have been shown to be required for or influence biofilm formation in both Gram-negative and Gram-positive bacteria but it would be interesting to know if there are bacteria that have no requirement for polyamines in planktonic growth and/or formation of biofilms.

\section{References}

Burrell M, Hanfrey CC, Murray EJ, Stanley-Wall NR, Michael AJ (2010) Evolution and multiplicity of arginine decarboxylases in polyamine biosynthesis and essential role in Bacillus subtilis biofilm formation. J Biol Chem 285: 39224-39238

Goytia M, Dhulipala VL, Shafer WM (2013) Spermine impairs biofilm formation by Neisseria gonorrhoeae. FEMS Microbiol Lett 343:64-69

Hamana K, Matsuzaki S (1992) Polyamines as a chemotaxonomic marker in bacterial systematics. Crit Rev Microbiol 18:261-283

Karatan E, Duncan TR, Watnick PI (2005) NspS, a predicted polyamine sensor, mediates activation of Vibrio cholerae biofilm formation by norspermidine. J Bacteriol 187: 7434-7443

Kolodkin-Gal I, Cao S, Chai L, Bottcher T, Kolter R, Clardy J, Losick R (2012) A self-produced trigger for biofilm disassembly that targets exopolysaccharide. Cell 149(3): 684-692

Lee J, Sperandio V, Frantz DE, Longgood J, Camilli A, Phillips MA, Michael AJ (2009) An alternative polyamine biosynthetic pathway is widespread in bacteria and essential for biofilm formation in Vibrio cholerae. J Biol Chem 284:9899-9907

McGinnis MW, Parker ZM, Walter NE, Rutkovsky AC, Cartaya-Marin C, Karatan E (2009) Spermidine regulates Vibrio cholerae biofilm formation via transport and signaling pathways. FEMS Microbiol Lett 299:166-174

Patel CN, Wortham BW, Lines JL, Fetherston JD, Perry RD, Oliveira MA (2006) Polyamines are essential for the formation of plague biofilm. J Bacteriol 188:2355-2363 
Sakamoto A, Terui Y, Yamamoto T, Kasahara T, Nakamura M, Tomitori H, Yamamoto K, Ishihama A, Michael AJ, Igarashi K, Kashiwagi K (2012) Enhanced biofilm formation and/or cell viability by polyamines through stimulation of response regulators UvrY and CpxR in the two-component signal transducing systems, and ribosome recycling factor. Int J Biochem Cell Biol 44:1877-1886
Wortham BW, Oliveira MA, Fetherston JD, Perry RD (2010) Polyamines are required for the expression of key Hms proteins important for Yersinia pestis biofilm formation. Environ Microbiol 12:2034-2047

Zhang X, Zhang Y, Liu J, Liu H (2013) PotD protein stimulates biofilm formation by Escherichia coli. Biotech Lett. doi: 10.1007/s10529-013-1184-8 